\title{
Tratamento Cirúrgico da Doença de Refluxo Gastroesofágico na Esclerose Sistêmica ${ }^{(*)}$
}

\section{Surgical Treatment of Gastroesophageal Reflux Disease in Systemic Sclerosis}

\author{
Percival D. Sampaio-Barros ${ }^{(1)}$, Luis Roberto Lopes ${ }^{(2)}$, Nelson Adami Andreollo ${ }^{(2)}$, André Gusmão Cunha ${ }^{(3)}$, \\ Adil Muhib Samara ${ }^{(4)}$, Nelson Ari Brandalise ${ }^{(5)}$ e João Francisco Marques Neto ${ }^{(4)}$
}

\section{RESUMO}

Objetivo: Os autores descrevem sua experiência com o tratamento cirúrgico da doença de refluxo gastroesofágico (DRGE) em pacientes com esclerose sistêmica (ES). Métodos: Foram selecionados 10 pacientes com DRGE que apresentavam esofagite grave e estenose esofágica, tratados previamente com doses recomendadas de drogas anti-secretórias (ranitidina e/ou omeprazol) e pró-cinéticas (cisapride) por mais de seis meses, sem melhora significativa. Todos os pacientes eram do sexo feminino e 8 eram caucasóides, sendo 7 com ES limitada e 3 com ES difusa. Resultados: $\mathrm{O}$ tratamento cirúrgico foi realizado através de videolaparoscopia em 9 pacientes e por cirurgia aberta no outro paciente. Sete pacientes foram submetidos à técnica de Nissen modificada e 3 à técnica de Lind. Seis pacientes com estenose esofágica significativa necessitaram de dilatações endoscópicas no período pré-operatório. Avaliação pós-operatória três meses após a cirurgia revelou que 70\% dos pacientes apresentaram resultado favorável, com melhora significativa da azia e da disfagia; 1 paciente necessitou de nova intervenção cirúrgica em consequiência de uma hérnia paraesofágica no período pós-operatório, sendo realizada uma gastrectomia em Y de Roux. Uma boa evolução foi referida por $80 \%$ dos pacientes um ano após cirurgia e por $70 \%$ dois anos após cirurgia, observando-se dois óbitos. Conclusões: Os autores concluem que o tratamento cirúrgico da DR GE representa uma eficiente opção terapêutica em pacientes com ES e esofagite grave com estenose.

Palavras-chave: esclerose sistêmica, doença de refluxo gastroesofágico, cirurgia.

\begin{abstract}
Objective: The authors describe the experience with the surgical treatment of gastroesophageal reflux disease (GERD) in 10 patients with systemic sclerosis (SSc). Methods: Criteria for surgery included GERD with severe chronic esophagitis and stricture, treated previously with recommended doses of antisecretory (ranitidine and/or omeprazole) and prokinetic (cisapride) drugs for more than six months, without significant improvement. All patients were female and 8 were Caucasian, with 7 of them presenting limited SSC and 3, diffuse SSC. Results: Surgical treatment was performed through videolaparoscopy in 9 patients and open surgery in 1 patient. Seven patients underwent modified Nissen technique and 3 patients, Lind technique. Significant esophageal strictures needed preoperative dilatations in 6 patients. Postoperative assessment three months after surgery revealed that $70 \%$ of the patients had a favourable outcome, with significant improvement in the heartburn and dysphagia; 1 patient needed a reoperation due to a paraesophageal hernia (Roux-en- $Y$ gastrectomy) in the postoperative period. A good outcome was referred by $80 \%$ of the patients one year after surgery and by $70 \%$ of them two years after surgery, occurring two deaths. Conclusions: The authors conclude that the surgical treatment of GERD represents an efficient therapeutic option in SSc patients with severe esophagitis and stricture.
\end{abstract}

Keywords: systemic sclerosis, gastroesophageal reflux disease, surgery.

\footnotetext{
* Trabalho realizado na Disciplina de Reumatologia do Departamento de Clínica Médica e na Disciplina de Gastroenterologia do Departamento de Cirurgia da Faculdade de Ciências Médicas da Universidade Estadual de Campinas (UNICAMP). Este trabalho contou com o apoio do Fundo de Amparo à Pesquisa da Sociedade Brasileira de Reumatologia. Recebido em 06/10/03. Aprovado, após revisão, em 16/01/04.

1. Assistente-Doutor da Disciplina de Reumatologia. UNICAMP.

2. Professor Assistente-Doutor da Disciplina de Gastroenterologia. UNICAMP.

3. Ex-Residente da Disciplina de Gastroenterologia. UNICAMP.

4. Professor Titular da Disciplina de Reumatologia. UNICAMP.

5. Professor Adjunto da Disciplina de Gastroenterologia. UNICAMP.
}

Endereço para correspondência: Dr. Percival D. Sampaio-Barros. Disciplina de Reumatologia, Departamento de Clínica Médica. Faculdade de Ciências Médicas - Universidade Estadual de Campinas (UNICAMP). Barão Geraldo, Campinas, SP, CEP 13081-970. E-mail: psbarros@fcm.unicamp.br 


\section{INTRODUÇÃO}

A esclerose sistêmica (ES) é uma doença difusa do tecido conjuntivo que afeta primariamente a pele e os órgãos internos. $\mathrm{O}$ acometimento do trato digestivo tem sido descrito em mais de $90 \%$ dos pacientes ${ }^{(1)}$ e o esôfago está freqüentemente afetado, apresentando desordens de motilidade em $75 \%$ a $90 \%$ dos $\operatorname{casos}^{(2)}$. A doença de refluxo gastroesofágico (DRGE) é uma complicação comum no paciente com ES, apresentando significativa morbidade. A fibrose da musculatura lisa dos dois terços inferiores do esôfago causando deficiência na peristalse, associada à diminuição na pressão de repouso do esfincter esofágico inferior (EEI), gera um constante refluxo de conteúdo gástrico para o esôfago ${ }^{(1)}$. Este refluxo crônico pode resultar em diversas complicações, incluindo esofagite, estenose, metaplasia de Barrett e neoplasia ${ }^{(3)}$.

O tratamento da DRGE na ES consiste em ajustes na dieta, uso de drogas anti-secretórias e pró-cinéticas, e cirurgia anti-refluxo ${ }^{(1)}$. Os agentes pró-cinéticos mais utilizados na ES são a metoclopramida ${ }^{(4)}$ e a cisaprida ${ }^{(5)}$, drogas que podem aumentar a pressão no EEI e também a amplitude das contrações peristálticas esofágicas. Contudo, descrições de arritmias cardíacas graves associadas ao uso da cisaprida fizeram com que a Food and Drug Administration nos Estados Unidos contra-indicasse o seu uso em pacientes que estivessem tomando medicações que pudessem prolongar o intervalo QT ou em pacientes com doença cardíaca prévia ou outras condições que pudessem predispor a uma arritmia cardíaca ${ }^{(6)}$.

Estudos recentes têm demonstrado que o tratamento cirúrgico da DRGE idiopática é realmente eficaz ${ }^{(7-10)}$, com poucas complicações ${ }^{(11)}$; e uma revisão concluiu que, em trabalhos avaliando DRGE crônica grave, a cirurgia pode se apresentar mais eficaz que o tratamento médico no alívio dos sintomas da esofagite, embora o uso do omeprazol possa acarretar alívio sintomático similar com o ajuste da dose $^{(12)}$. O tratamento cirúrgico da DRGE na ES está ainda limitado a alguns relatos de caso ${ }^{(13-18)}$, mas pode vir a representar uma importante opção terapêutica no manejo de pacientes esclerodérmicos com sintomas esofágicos graves não responsivos ao tratamento convencional.

Os autores apresentam sua experiência com o tratamento cirúrgico (com um seguimento de dois anos) de 10 pacientes com ES e DRGE grave, baseado numa boa evolução em $93 \%$ de 59 pacientes não esclerodérmicos com DRGE previamente submetidos a cirurgia videolaparoscópica no Hospital de Clínicas da Unicamp ${ }^{(19)}$.

\section{PACIENTES E MÉTODOS}

O presente estudo analisa a evolução de 10 pacientes com ES submetidos a tratamento cirúrgico para DRGE. Estes pacientes representaram 5\% dos 202 pacientes com ES atendidos no ambulatório de Esclerodermia do Hospital de Clínicas da Unicamp no período 1991-2000, e apresentavam DRGE com esofagite crônica grave e estenose. Todos os pacientes preenchiam os critérios para ES do Colégio Americano de Reumatologia ${ }^{(20)}$, e foram subdivididos em ES limitada e ES difusa de acordo com os critérios de LeRoy et al. ${ }^{(21)}$. Todos os pacientes eram do sexo feminino e 8 eram caucasóides, sendo 7 com ES limitada (com média de idade 53 anos e média de duração de doença 17 anos) e 3 com ES difusa (com média de idade 44 anos e média de duração da doença seis anos).

O acometimento esofágico nos pacientes esclerodérmicos submetidos à cirurgia anti-refluxo foi determinado pelo esofagograma contrastado (analisando hipomotilidade e dilatação esofágicas, hérnia hiatal e DRGE), manometria esofágica (analisando distúrbios da motilidade esofágica e a pressão de repouso do EEI), endoscopia digestiva alta (avaliando esofagite, estenose e esôfago de Barrett) e cintilografia esofágica (avaliando o tempo de clareamento esofágico e confirmando o refluxo gastroesofágico). Todos os pacientes foram tratados previamente com doses recomendadas de drogas anti-secretórias (ranitidina e/ou omeprazol) e pró-cinéticas (cisaprida) por período mínimo de seis meses, sem melhora significativa. Seis pacientes necessitaram de dilatações endoscópicas préoperatórias em razão da estenose esofágica.

Duas técnicas cirúrgicas, via videolaparoscopia, foram utilizadas neste estudo, a técnica de $\operatorname{Lind}^{(22)}$ e a técnica de Nissen ${ }^{(23)}$ modificada; a descrição completa da técnica cirúrgica de Nissen modificada pode ser conferida no trabalho de Lopes et al. ${ }^{(19)}$.

A avaliação clínica pré-operatória foi comparada à avaliação pós-operatória utilizando-se a classificação de Visick modificada ${ }^{(24)}$ : grau I - evolução excelente, sem sintomas; grau II - boa evolução, com sintomas; grau III - evolução satisfatória, com sintomas moderados não controlados pela dieta, causando algum desconforto; grau IV - evolução não satisfatória. A avaliação pós-operatória foi realizada três meses, um ano e dois anos após a cirurgia. Os pacientes também foram submetidos a uma avaliação endoscópica três meses após a cirurgia. As endoscopias digestivas altas foram graduadas de acordo com os critérios de Savary-Miller ${ }^{(25)}$. 


\section{RESULTADOS}

No período pré-operatório, os pacientes queixavam-se de azia e disfagia significativas, com regurgitação em dois casos. Manometria esofágica pré-operatória demonstrou hipomotilidade esofágica ou aperistalse com uma pressão de repouso em EEI baixa (média $4.9 \pm 1.2 \mathrm{mmHg}$; normal = $10-23 \mathrm{mmHg}$ ). Cintilografia esofágica revelou um tempo de clareamento esofágico prolongado (média 50 segundos; normal $=10 \pm 2$ segundos). Seis pacientes com estenose esofágica diagnosticada via esofagograma contrastado e endoscopia digestiva foram submetidos a dilatações endoscópicas pré-operatórias. Uma paciente teve diagnóstico de esôfago de Barrett, confirmado por biópsia, e foi submetida a ablação com laser no período pré-operatório.

A correção cirúrgica da DRGE foi realizada por via videolaparoscópica em 9 pacientes. Uma paciente com ES limitada apresentando aperistalse esofágica, estenose significativa e esofagite grave foi submetida a cirurgia aberta. Sete pacientes realizaram a técnica de Nissen modificada e 3 a técnica de Lind. Uma paciente com ES limitada apresentou hérnia paraesofágica com migração da válvula para o tórax e recorrência dos sintomas no período pós-operatório imediato, necessitando de uma ressecção gástrica com reconstrução em Y de Roux; esta paciente apresentou candidíase esofágica e vômitos no mesmo período. Não houve outras complicações cirúrgicas ou mortes relacionadas à cirurgia.

Avaliação clínica pós-operatória após três meses revelou que $70 \%$ dos pacientes apresentaram uma evolução favorável, com 5 pacientes apresentando Visick grau I e 2 Visick grau II. Endoscopia pós-operatória realizada naqueles 6 pacientes com estenose esofágica e necessidade prévia de dilatações não evidenciou recidiva da estenose. Os achados endoscópicos nos períodos pré e pós-operatório e a classificação de Visick estão descritos na Tabela 1.

Oito pacientes receberam alta hospitalar três dias após a cirurgia. A paciente que necessitou uma cirurgia aberta permaneceu hospitalizada por oito dias, enquanto que a paciente que apresentou migração da válvula recebeu alta após 17 dias de internação.

Um ano após a cirurgia anti-refluxo, foi observada uma boa evolução em $80 \%$ dos casos, com 5 pacientes apresentando Visick grau I e 3 Visick grau II; 5 pacientes (aqueles com Visick graus II, III e IV) tiveram a necessidade de usar droga pró-cinética (cisaprida) após a cirurgia. Dois anos após a cirurgia, 2 pacientes com DRGE grave e estenose haviam falecido, em conseqüência do envolvimento fibrótico de múltiplos órgãos; entre os 8 pacientes restantes, 7 referiram uma boa evolução (Tabela 1).
TABELA 1

Resultados De endoscopia digestiva alta (anTes E APÓs CIRURGIA ANTI-REFLUXO) E CLASSIFICAÇÃO DE VISICK

\begin{tabular}{lccccc}
\hline Endoscopia & Dilatações & Endoscopia & \multicolumn{3}{c}{ Visick } \\
Pré-0peratória & Pré-0peratórias & Pós-Operatória & 3m & 1a & 2a \\
\hline G III & Não & Normal & I & I & I \\
G IV + estenose & 5 sessões & Normal & I & I & I \\
G IV + estenose & 1 sessão & G I & I & I & - \\
G IV + estenose & 9 sessões & Normal & I & I & I \\
G III & Não & Normal & I & I & I \\
G IV + estenose & 2 sessões & Normal & II & II & II \\
G I & Não & Normal & II & II & II \\
G IV + estenose & 1 sessão & G IV & III & II & II \\
G IV + estenose & 3 sessões & Normal & III & III & - \\
G II & Não & G III & IV & IV & IV \\
\hline
\end{tabular}

* Resultados de endoscopia digestiva alta graduados de acordo com os critérios de Savary-Miller ${ }^{(25)} ; 3 \mathrm{~m}=$ três meses após cirurgia; $1 \mathrm{a}=u \mathrm{~m}$ ano após cirurgia; $2 \mathrm{a}=$ dois anos após cirurgia; - = óbito.

\section{DISCUSSÃO}

Como o acometimento esofágico é bastante freqüente nas duas formas clínicas da ES, é importante ter-se em mente que um tratamento adequado e precoce, com drogas prócinéticas e anti-secretórias, faz-se necessário quando se pretende evitar complicações graves como estenose, esôfago de Barrett e adenocarcinoma. $\mathrm{O}$ envolvimento esofágico pode se manifestar com uma progressão muito lenta, como em alguns pacientes com ES limitada de longa duração ${ }^{(1)}$, ou pode expressar um envolvimento orgânico grave precoce em pacientes com ES difusa ${ }^{(26)}$. No presente estudo, DRGE grave e requerendo tratamento cirúrgico foi observada em pacientes com ES limitada com doença de longa duração (média 17 anos) e também em pacientes com ES difusa com doença de curta duração (média 6 anos). Acometimento esofágico foi observado em $86.7 \%$ dos pacientes esclerodérmicos na experiência dos autores, cursando com significativa morbidade ${ }^{(27)}$

Recomendações quanto à ressecção esofágica ${ }^{(28)}$, receio na confecção de uma válvula num esôfago hipotônico ${ }^{(17)} \mathrm{e}$ a falta de dados científicos objetivos levaram a maioria dos cirurgiões gástricos a evitar a cirurgia anti-refluxo em pacientes com ES. A evolução a curto prazo com a ressecção esofágica apresenta uma morbidade significativa, a despeito da melhora clínica ${ }^{(29,30)}$. A experiência prévia dos autores incluiu três pacientes com ES e estenose esofágica que evoluíram para esofagectomia (duas reconstruções de tubo digestivo e uma interposição de alça jejunal, pela técnica 
de Merendino) e mostraram recorrência dos sintomas durante o seguimento, em razão da estenose da anastomose.

Os primeiros trabalhos demonstrando a evolução da cirurgia anti-refluxo na ES mostraram uma significativa melhora clínica em $69 \%$ a $75 \%$ dos pacientes ${ }^{(14-16)}$. Mais recentemente, Poirier et al. ${ }^{(18)}$ descreveram regressão da estenose péptica em 6 de 7 pacientes (85\%); nenhum destes pacientes foi submetido a dilatação esofágica antes da cirurgia. Por outro lado, Mansour e Malone ${ }^{(17)}$ realizaram cirurgia anti-refluxo em 12 pacientes esclerodérmicos, com recorrência dos sintomas em todos eles; 7 pacientes necessitaram reoperações ou dilatações periódicas.

As técnicas de cirurgia anti-refluxo devem seguir alguns princípios básicos para serem eficazes, restaurando a competência do cárdia ${ }^{(31)}$ e preservando o clareamento esofágico ${ }^{(32)}$. No presente estudo, optou-se pelo uso da técnica de Nissen modificada, que combina elementos das técnicas de Nissen e Lind, contribuindo para aumentar a competência da válvula, com menor número de efeitos colaterais. A técnica original de Nissen criava uma fundoplicatura utilizando-se a parede gástrica posterior sobre o esôfago abdominal ${ }^{(23)}$; modificações importantes foram o estreitamento da válvula e a construção de uma válvula livre de tensão, contribuindo para diminuir a recorrência de disfagia sem perder a habilidade anti-refluxo. Neste estudo, uma significativa melhora clínica $(70 \%$, incluindo Visick I e II) foi observada no período pós-operatório imediato, com permanência da boa evolução um e dois anos após a cirurgia. Seis pacientes que apresentaram estenose esofágica previamente à cirurgia anti-refluxo e necessitaram dilatações endoscópicas não apresentaram estenose no período pós-operatório. Epitélio colunar metaplásico é freqüente na DRGE por causa da ES, podendo ocorrer em um terço dos pacientes ${ }^{(2)}$, e o tratamento cirúrgico não interfere com sua permanência. Sendo assim, nossa escolha de tratamento no único caso de esôfago de Barrett foi ablação a laser após a fundoplicatura laparoscópica.

A função esofágica parece não ser afetada pela fundoplicatura. Poirier et al. ${ }^{(18)}$ realizaram estudo cintilográfico nos períodos pré e pós-operatório, e não observaram diminuição significativa do clareamento esofágico; estudos manométricos não evidenciaram alterações significativas na contratilidade esofágica, enquanto era observado um aumento na pressão de repouso do EEI, de 10 para 14 $\mathrm{mmHg}$. Wetscher et al. ${ }^{(33)}$, analisando 24 pacientes, demonstraram que uma fundoplicatura parcial pode aumentar a função de clareamento esofágico em pacientes com distúrbios da motilidade relacionados à DRGE, associado à melhora clínica na disfagia; nenhum de seus pacientes apresentava esclerodermia. Por outro lado, Fibbe et al. ${ }^{(34)}$, estudando 200 pacientes estratificados de acordo com a presença ou ausência de distúrbio de motilidade esofágica (100 pacientes por grupo), concluíram que o distúrbio de motilidade esofágica era ocasionado por uma doença mais agressiva e que não seria corrigido pela fundoplicatura, independente do procedimento executado.

Em termos de relação risco/benefício, o risco de uma evolução desfavorável após uma cirurgia laparoscópica é maior que aquele observado com o tratamento de manutenção com os inibidores da bomba protônica, tornando necessária uma seleção cuidadosa dos pacientes a serem considerados para cirurgia ${ }^{(35,36)}$. A evolução dos pacientes selecionados para cirurgia anti-refluxo tem demonstrado melhora na qualidade de vida no seguimento a longo prazo $^{(9)}$, e quando comparado ao tratamento não cirúrgico $^{(37,38)}$. No entanto, como a cirurgia anti-refluxo não é isenta de complicações, os pacientes devem ser informados que a reoperação por via laparoscópica após um procedimento anti-refluxo sem sucesso se constitui num dificil desafio cirúrgico não isento de significativa morbidade ${ }^{(39)}$. Henderson et al. ${ }^{(40)}$, analisando 100 pacientes com estenose esofágica tratados cirurgicamente, identificaram três características associadas a um pior prognóstico: cirurgia prévia, estenose grave e esclerodermia. Quando não havia nenhuma destas características, a construção da válvula antirefluxo apresentava uma boa evolução a longo prazo; contudo, quando duas ou três destas características estavam presentes, o prognóstico era realmente ruim.

Desta forma, o tratamento precoce do acometimento esofágico, visando prevenir a evolução para uma esofagite grave com estenose, é condição indispensável para se prevenir uma significativa morbidade esofágica em pacientes esclerodérmicos. A conduta inicial num paciente com ES e acometimento esofágico (hipomotilidade e/ou DRGE de qualquer intensidade) é prescrever tratamento com drogas antisecretórias (preferencialmente omeprazol) e pró-cinéticas (cisaprida), associado à orientação nutricional, a fim de evitar que o freqüente refluxo gastroesofágico que ocorre nos pacientes esclerodérmicos se converta numa esofagite com estenose grave. Com as recentes considerações sobre o uso da cisaprida $^{(6)}$, novas drogas pró-cinéticas com melhor perfil de segurança necessitam ser desenvolvidas. A opção cirúrgica para a DRGE deve ser considerada somente após a não resposta à terapêutica convencional (com duração mínima de seis meses), quando o paciente mantém significativa sintomatologia clínica aliada à qualidade de vida ruim. A análise de custos capacitando 
o clínico a escolher o tratamento mais eficiente e custo/efetivo para a DRGE é outra consideração importante ${ }^{(41,42)}$, mas no momento não existem estudos avaliando especificamente pacientes com ES.

\section{REFERÊNCIAS}

1. Weinstein WM, Kadell BM: The gastrointestinal tract in systemic sclerosis. Em Clements PJ, Furst DE (Editors): Systemic sclerosis. $2^{\mathrm{a}}$ edição, Filadélfia, Lippincott Williams \& Wilkins, pg. 293-308, 2004.

2. Sjogren RW: Gastrointestinal motility disorders in scleroderma. Arthritis Rheum 37: 1265-82, 1994.

3. Fitzgerald RC, Triadafilopoulos G: Esophageal manifestations of rheumatic diseases. Semin Arthritis Rheum 26: 641-56, 1997.

4. Johnson DA, Drane WE, Curran J, et al: Metoclopramide response in patients with progressive systemic sclerosis: effect on esophageal and gastric motility abnormalities. Arch Intern Med 147: 1597-601, 1987.

5. Horowitz M, Maddern GJ, Maddox A, Wishart J, Chatterton BE, Shearman DJC: Effects of cisapride on gastric and esophageal emptying in progressive systemic sclerosis. Gastroenterology 93: 3115, 1987.

6. Smalley W, Shatin D, Wysowski DK, et al: Contraindicated use of cisapride: impact of Food and Drug Administration Regulatory Action. JAMA 284: 3036-9, 2000.

7. Bais JE, Bartelsman JFWM, Bonjer HJ, et al: Laparoscopic or conventional Nissen fundoplication for gastro-oesophageal reflux disease: randomized clinical trial. Lancet 355: 170-4, 2000.

8. Lafullarde T, Watson DI, Jamieson GG, Myers JC, Game PA, Devitt P: Laparoscopic Nissen fundoplication: five-year results and beyond. Arch Surg 136: 180-4, 2001.

9. Bammer T, Hinder RA, Klaus A, Klingler PJ: Five-to-eight-year outcome of the first laparoscopic Nissen fundoplications. J Gastrointest Surg 5: 42-8, 2001

10. Spechler SJ, Lee E, Ahnen D, et al: Long-term outcome of medical and surgical therapies for gastroesophageal reflux disease: follow-up of a randomized controlled trial. JAMA 285: 2331-8, 2001.

11. Pohl D, Eubanks TR, Omelanczuk PE, Pellegrini CA: Management and outcome of complications after laparoscopic antireflux operations. Arch Surg 136: 399-404, 2001.

12. Allgood PC, Bachmann M: Medical or surgical treatment for chronic gastroesophageal reflux? A systematic review of published evidence of effectiveness. Eur J Surg 166: 713-21, 2000.

13. McLaughlin JS, Roig R, Woodruff MFA: Surgical treatment of strictures of the esophagus in patients with scleroderma. J Thorac Cardiovasc Surg 64:641-5, 1971.

14. Henderson RD, Pearson FG: Surgical management of esophageal scleroderma. J Thorac Cardiovasc Surg 66: 686-92, 1973.

15. O'Leary JP, Hollenbeck JI, Woodward ER: Surgical treatment of esophageal stricture in patients with scleroderma. Am Surg 41: 131-5, 1975.

16. Orringer MB, Orringer JS, Dabich L, Zarafonetis JD: Combined Collis gastroplasty-fundoplication operations for scleroderma reflux esophagitis. Surgery 90: 624-30, 1981.
Concluindo, o tratamento cirúrgico da DRGE por via videolaparoscópica parece representar uma eficiente opção terapêutica em pacientes com ES cuidadosamente selecionados, com esofagite e estenose graves.

17. Mansour KA, Malone CE: Surgery for scleroderma of the esophagus: a 12-year experience. Ann Thorac Surg 46: 513-4, 1988.

18. Poirier NC, Taillefer R, Topart P, Duranceau A: Antireflux operations in patients with scleroderma. Ann Thorac Surg 58: 66-73, 1994.

19. Lopes LR, Brandalise NA, Andreollo NA, Leonardi LS: Tratamento cirúrgico videolaparoscópico da doença do refluxo gastroesofagiano: técnica de Nissen modificada - resultados clínicos e funcionais. Rev Ass Med Brasil 47:141-8, 2001.

20. Subcommittee for Scleroderma Criteria of the American Rheumatism Association Diagnostic and Therapeutic Criteria Committee: Preliminary criteria for the classification of systemic sclerosis (scleroderma). Arthritis Rheum 23: 581-90, 1980.

21. LeRoy EC, Black C, Fleischmajer R, et al: Scleroderma (systemic sclerosis): classification, subsets and pathogenesis. J Rheumatol 15: 202-5, 1988.

22. Lind JF, Burns CM, McDougall C: "Physiological" repair for hiatus hernia - manometric study. Arch Surg 91: 233-7, 1965.

23. Nissen R: Gastropexy as the lone procedure in the surgical repair of hiatus hernia. Am J Surg 92: 389-92, 1956.

24. Goligher JC, Pulvercraft CN, Dombal FT, et al: Five to eight year results of Leeds/York controlled trial of elective surgery for duodenal ulcer. Br Med J ii: 781-7, 1968.

25. Savary M, Miller G: The oesophagus. Em Gasmann SA, editor: Handbook and atlas of endoscopy. Solothurn, Verlag Gossman, pg. 135-42, 1978.

26. Steen VD, Medsger TA Jr: Severe organ involvement in systemic sclerosis with diffuse scleroderma. Arthritis Rheum 43:2437-44, 2000.

27. Sampaio-Barros PD, Barcelos IK, Carvalho ACO, Samara AM, Marques Neto JF: Acometimento do trato digestivo na esclerose sistêmica. Rev Bras Reumatol 39: 81-6, 1999.

28. Bonavina L, Fontebasso V, Bardini R, Baessato M, Perachia A: Surgical treatment of reflux stricture of the oesophagus. Br J Surg 80: 317-20, 1993.

29. Topart P, Deschamps C, Taillefer R, Duranceau A: Sclerodermie et reflux oesophagien: controle chirurgical. Ann Chir 46: 794-9, 1992.

30. Peix JL, Maroun J, Tekinel O: Traitement de l'oesophagite sclerodermique: interet de la diversion duodenale. Ann Chir 47: 302-6, 1993.

31. Patti MG, Gantert W, Way LW: Surgery of the esophagus: anatomy and physiology. Surg Clin North Am 77: 959-70, 1997.

32. Horgan S, Pellegrini CA: Surgical treatment of gastroesophageal reflux disease. Surg Clin North Am 77: 1063-82, 1997.

33. Wetscher GJ, Glaser K, Gadenstatter M, Wieschemeyer T, Profanter $\mathrm{C}$, Klinger P: Laparoscopic partial posterior fundoplication improves poor oesophageal contractility in patients with gastrooesophageal reflux disease. Eur J Surg 164: 679-84, 1998.

34. Fibbe C, Layer P, Keller J, Strate U, Emmermann A, Zornig C: Esophageal motility in reflux disease before and after fundoplication: a prospective, randomized, clinical, and manometric study. Gastroenterology 121: 214-6, 2001. 
35. Glaser K, Wetscher GJ, Klingler A, et al: Selection of patients for laparoscopic antireflux surgery. Dig Dis 18: 129-37, 2000.

36. Kahrilas PJ: Management of GERD: medical versus surgical. Semin Gastrointest Dis 12: 3-15, 2001.

37. Slim K, Bousquet J, Kwiatkowski F, Lescure G, Pezet D, Chipponi $\mathrm{J}$ : Quality of life before and after laparoscopic fundoplication. Am J Surg 180: 41-5, 2000.

38. Fernando HC, Schauer PR, Rosenblatt M, et al: Quality of life after antireflux surgery compared with nonoperative management for severe gastroesophageal reflux disease. J Am Coll Surg 194: 23-7, 2002.
39. Neuhauser B, Hinder RA: Laparoscopic reoperation after failed antireflux surgery. Semin Laparosc Surg 8: 281-6, 2001.

40. Henderson RD, Henderson RF, Marryatt GV. Surgical management of 100 consecutive esophageal strictures. J Thorac Cardiovasc Surg 99: 1-7, 1990.

41. O'Connor JB, Provenzale D, Brazer S: Economic considerations in the treatment of gastroesophageal reflux disease: a review. Am J Gastroenterol 95: 3356-64, 2000.

42. Johnson DA: Endoscopic therapy for GERD- Baking, sewing, or stuffing: An evidence-based perspective. Rev. Gastroenterol Disord 3: 142-9, 2003. 